\title{
Wireless Capsule Enteroscopy in Healthy Volunteers
}

\author{
Ilja Tachecí,", Petr Bradna ${ }^{1}$, Tomáš Douda ${ }^{1}$, Drahomíra Baštecká ${ }^{1}$, Marcela Kopáčová ${ }^{1}$, \\ Stanislav Rejchrt ${ }^{1}$, Martin Lutonskýz, Tomáš Soukup ${ }^{1}$, Jan Bureš ${ }^{1}$
}

\begin{abstract}
${ }^{1}$ Second Department of Internal Medicine - Gastroenterology; Charles University, Faculty of Medicine and University Hospital, Hradec Králové, Czech Republic

${ }^{2}$ Department of Orthopedic Surgery; Charles University, Faculty of Medicine and University Hospital, Hradec Králové, Czech Republic

* Corresponding author: Second Department of Internal Medicine - Gastroenterology, Charles University, Faculty of Medicine, Sokolská 581, 500 05, Hradec Králové, Czech Republic; tacheci@gmail.com
\end{abstract}

Summary: Introduction: The aim of our prospective study was to define endoscopy appearance of the small bowel in healthy volunteers. Method: Forty-two healthy volunteers underwent wireless capsule endoscopy, clinical investigation, laboratory tests, and completed a health-status questionnaire. All subjects were available for a 36-month clinical follow-up. Results: Eleven subjects (26\%) had fully normal endoscopy findings. Remaining 31 persons (74\%), being asymptomatic, with normal laboratory results, had some minor findings at wireless capsule endoscopy. Most of those heterogeneous findings were detected in the small intestine $(27 / 31 ; 87 \%)$, like erosions and/or multiple red spots, diminutive polyps and tiny vascular lesions. During a 36-month clinical follow-up, all these 42 healthy volunteers remained asymptomatic, with fully normal laboratory control. Conclusions: Significant part of healthy subjects had abnormal findings at wireless capsule endoscopy. These findings had no clinical relevance, as all these persons remained fully asymptomatic during a 36-month follow-up. Such an endoscopic appearance would be previously evaluated as "pathological". This is a principal report alerting that all findings of any control group of wireless capsule endoscopic studies must be evaluated with caution.

Keywords: Small bowel; Wireless capsule enteroscopy; Healthy volunteers; 36-month follow-up

\section{Introduction}

Wireless capsule endoscopy represents an important progress in small intestinal imaging. During past 16 years (since 2000 (1)) it has become the leading enteroscopy method. It is now commonly used in the evaluation of obscure gastrointestinal bleeding (including iron deficiency anaemia), suspected and known Crohn's disease, small bowel tumours, complicated coeliac disease and non-steroidal anti-inflammatory drug-induced enteropathy.

The main advantage of the method is its minimal invasiveness, safety and reliability. That is why capsule endoscopy is often used both in daily clinical practice and in clinical studies on small bowel disease. Thanks to its high sensitivity capsule endoscopy is able to identify small lesions with little or no clinical significance, too. Usually, there is no problem in identifying small bowel tumours, sources of bleeding and advanced inflammatory changes (ulcers, cobble stones, inflammatory polyps, multiple erosions and aphthae). However, interpreting of tiny non-specific findings suggestive of inflammation (swelling, redness, isolated erosions) is often questionable. Mild non-specific inflammatory-like lesions were frequently described in the small bowel of healthy volunteers in many capsule endoscopy studies as pathologic ones. We found this type of lesions in $12 \%$ of a control group in our study with non-steroidal anti-inflammatory drug-induced enteropathy (2), too.

From this perspective, it is necessary to identify and define the spectrum of normal or insignificant enteroscopy findings, and consider these findings in evaluating wireless capsule endoscopy studies. The aim of this study was to assess capsule endoscopy in healthy volunteers.

\section{Methods}

\section{Participants and design}

We organized our study as prospective one and it included adult ( $>18$ years) healthy volunteers. Exclusion criteria were any acute or chronic disease and history of use of any drugs (including non-steroidal anti-inflammatory drugs) in a month prior the study and pregnancy. Written informed consent was obtained from all participants.

A total of 42 healthy participants ( 29 women, mean age 42 years, median 42 years; 13 men, mean age 43 years, median 42 years) were enrolled in the study. All underwent 
wireless capsule endoscopy, basic clinical investigation, laboratory tests (comprising anaemia, nutrition and inflammatory markers: blood count, reticulocytes, Coombs test, serum iron, ferritin, albumin and prealbumin, C-reactive protein, erythrocyte sedimentation rate, Helicobacter pylori stool antigen and faecal occult blood testing). All subjects completed a health-status questionnaire.

Wireless capsule endoscopy was performed in compliance with the published guidelines of the Czech Society of Gastroenterology and European Society of Gastrointestinal Endoscopy $(3,4)$. The EndoCapsule ${ }^{\mathrm{TM}}$ (Olympus) was used. The participants were prepared by the 12-hour fasting only, without the need of any purgative solutions. 2 and 4 hours after the capsule ingestion the subjects were allowed fluids and light meal. The position of capsule endoscope was controlled 2 hours after its ingestion using a real time viewer. Two experienced endoscopists evaluated capsule recording. Any particular findings (regardless of clinical significance or insignificance) were identified as abnormal and were described according to the CEST (Capsule Endoscopy Standard Terminology) $(5,6)$. Red spot was defined as a demarcated, circular, reddish, small, pinpoint mucosal mark, erosion as a superficial destruction of mucosa, denuded areas as mucosal surface without villi, aphthous lesion as a mucosal break with a pale centre and reddish halo and ulcer as an excavated lesion with its base covered with fibrin. Vascular abnormalities were lymphangiectasias (whitish dots involving individual oedematous villi), angiectasias (flat, reddish or fernlike ectatic vessels) and flebectasias (large, ectatic, bluish veins). Basic time characteristics of the investigation (gastric transit time, small bowel transit time, total time of batteries function, time of findings) were evaluated, too. Small bowel visibility, based on the degree of purification of the intestine, was scored.

\section{Ethical approval}

All procedures were in accordance with the ethical standards of the institutional research committee and with the 1964 Helsinki declaration and its latter amendments.

\section{Results}

\section{Endoscopy findings}

There was no clinical or technical complication observed during the study. Capsule endoscopes reached the small bowel within the first 2 hours in all healthy volunteers and completed panenteroscopy before the batteries discharge. The quality of the small intestinal mucosa visibility was good in majority of investigations; in one participant only $(2.4 \%)$ the visibility was decreased due to the rich bowel content in some parts of distal ileum.

The average gastric transit time was $37 \pm 33$ minutes (median 27 minutes) and small bowel transit time was $262 \pm 90$ minutes (median 246 minutes). The total time of investigation (the battery function time) was $557 \pm 93$ minutes (median 571 minutes) on average. The mean evaluation time (time spent by an endoscopist to evaluate the completerecord) was $58 \pm 6$ minutes (median 55 minutes).

Eleven subjects $(26 \%)$ had fully normal endoscopy findings. Abnormal findings were identified in 31/42 subjects (74\%). The majority of these findings was described in the small bowel (27/42 persons; 64\%): isolated red spots (14/42; $33 \%)$, multiple red spots $(3 / 42 ; 7 \%)$, erosions $(2 / 42 ; 5 \%)$, mucosal erythema in the duodenal bulb classified as bulbitis $(4 / 42 ; 10 \%)$, suspected diminutive polyps $(3 / 42 ; 7 \%)$, small submucosal tumour, probably a tiny lipoma (1/42) and vascular findings like angiectasias (1/42), lymphangiectasias $(1 / 42)$ and small bowel flebectasia (1/42), small intestinal xanthomas $(2 / 42 ; 5 \%)$. Extraintestinal findings comprised reflux oesophagitis (1/42), the endoscopic picture of erythematous gastritis $(8 / 42 ; 19 \%)$, diminutive gastric polyps $(2 / 42 ; 5 \%)$, and erosions $(3 / 42 ; 7 \%)$. Small intestinal lesions were localised more frequently in the jejunum (16), the duodenal and ileal involvement was presented less commonly. Caecal polyp was identified in one subject. This person underwent subsequent colonoscopy with polypectomy of 4 adenomas with low-grade dysplasia. All other findings at wireless capsule endoscopy were considered as fully benign, requiring no further action.

\section{Clinical data}

All healthy volunteers were without any clinical symptoms and health problems before the inclusion and during the study period. Four people mentioned dypepsia in the past history (without need for treatment and without any other clinical consequences). All study participants were available for a 36-month clinical follow-up.

\section{Laboratory tests}

Laboratory tests were fully normal in the majority of subjects before wireless capsule endoscopy. The exception was significant increase in CRP $(>10 \mathrm{mg} / \mathrm{l})$, we found in three persons (11 mg/l, $12 \mathrm{mg} / \mathrm{l}$, and $22 \mathrm{mg} / \mathrm{l})$ during the initial examination. There was no significant capsule endoscopy finding in all these subjects (normal finding or isolated red spot in duodenum and ileum). There was no infectious complication subsequently diagnosed in them and control CRP levels normalized. Laboratory tests were normal after the 36-month follow-up.

\section{Discussion}

Our capsule endoscopy study revealed abnormal small intestinal findings in healthy volunteers that would be previously considered as minor but pathological ones and thus could be misinterpreted. All subjects were health professionals from our University Hospital and they were available for subsequent 36-month follow-up. All remained symp- 

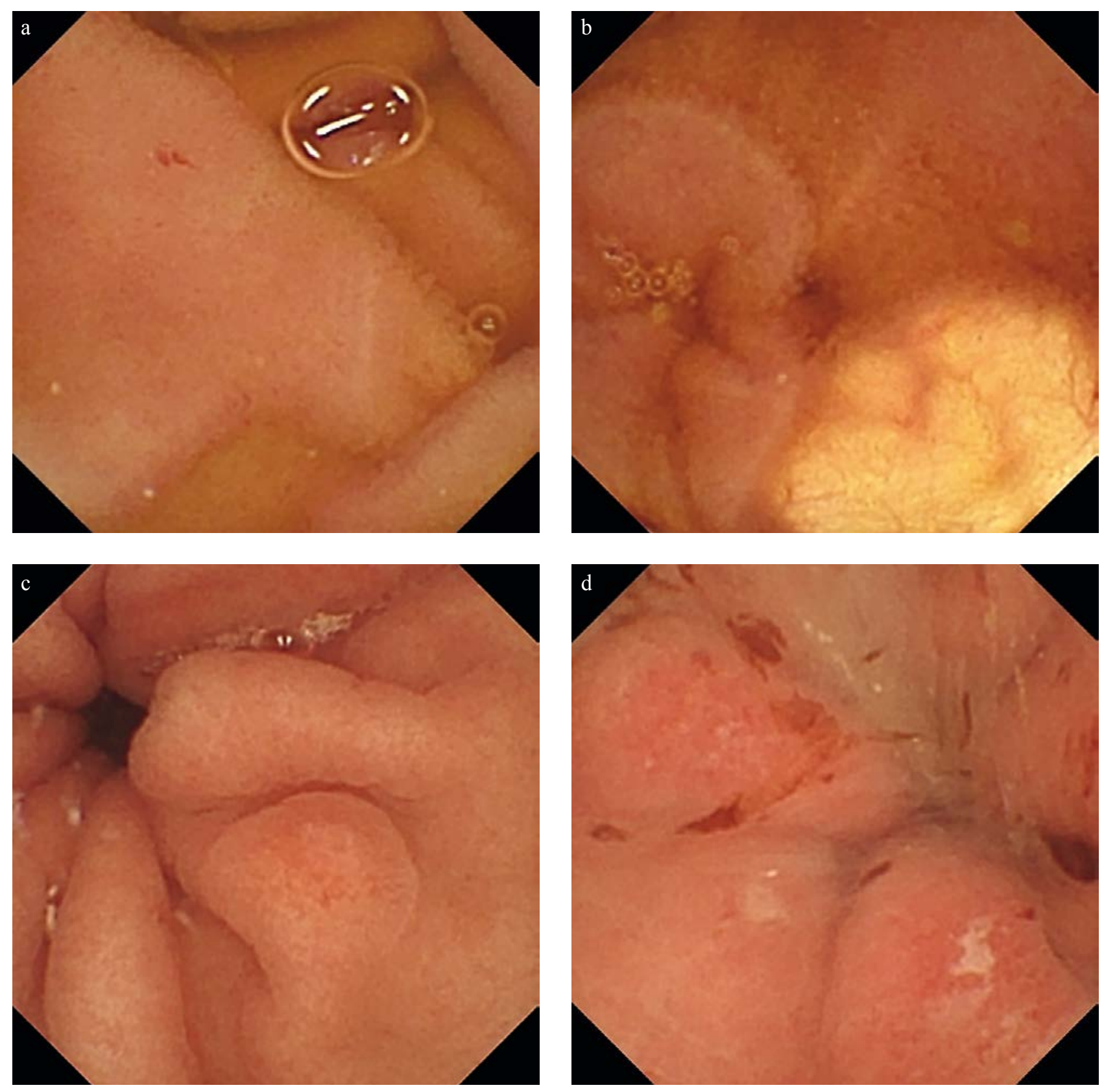

Fig. 1: CE findings: a) isolated red spot: jejunum; b) lipoma: jejunum; c) erosions: stomach; d) haemorrhagic erosions: stomach.

tom-free and had fully normal laboratory control by the end of the 36-month follow-up. Only 11 persons $(26 \%)$ had fully normal finding at capsule endoscopy. The key and most important finding of our study is the high prevalence (43\%) of so-called red spots in the small intestinal mucosa of healthy volunteers recognized by means of wireless capsule endoscopy.

Red spots of the small bowel were defined as demarcated, usually circular, 1-3 $\mathrm{mm}$ area of crimson mucosa with preservation of overlying villi and were described during the first enteroscopy procedures using sonde enteroscope (7). They were considered to be initial mucosal lesions that might progress into erosions or ulcers. This point of view has been repeatedly published without clear evidence of its basis. This terminology has been subsequently adopted by wireless capsule enteroscopy, but the definition of these lesions has been extended to any flat redness mucosa of a millimeter size, without any damage of the surface structure. Already early publications of capsule endoscopy warned against exaggerated interpretations of red spots as obvious 
pathology (8). Nevertheless, red spots were believed to be a potential source of bleeding (as resembling endoscopic image of angiectasias) in some papers and therefore these studies were evaluated as positive for significant findings (9). Some authors also considered red spots as the "pre-aphthoid phase" of typical mucosal lesions in Crohn's disease (10). Graham et al. included red spots into the scoring system of non-steroidal anti-inflammatory drug-induced lesions (11), followed by Maiden et al. (12) and Caunedo-Álvarez et al. (13). These findings were repeatedly described in different control groups and/or placebo users of these studies in different rates $(7-41 \%)(14,11,15)$. The explanation could be in overestimation of tiny mucosal lesions and terminological confusion that currently persists in the descriptions of capsule endoscopy. Wireless capsule endoscopy belongs to the most sensitive endoscopy methods with high diagnostic yield and the ability to identify diminutive, small bowel findings (optical system of currently used capsule endoscopes are theoretically able to identify objects less than $0.1 \mathrm{~mm}$ ) Our results indicate, that true red spots (pinpoint red mucosal marks) are non-specific, clinically non-significant lesions, which can be (if isolated or infrequent) regarded as normal and should not be confused with diagnostic findings. It should be clearly distinguished of typical angiectasias, which are small (2 to $10 \mathrm{~mm}$ ), flat, red lesions with a fernlike pattern of arborizing, ectatic vessels radiating from a central vessel (5).

Lesions identified by means of capsule endoscopy in our study can be divided from a clinical point of view into the two main groups: findings with a possible clinical potential and clinically non-significant objects. Angiectasias, bulbitis and erosions can be included into the first one. Small bowel angiectasias are the most common vascular anomalies found within the gastrointestinal tract especially in the older patients. Although it can cause obscure gastrointestinal bleeding, angiectasias are often diagnosed incidentally and remains clinically silent for many years; the indication for treatment is bleeding or iron deficiency anaemia. We identified small typical angiectasias in the jejunum and ileum of a 51-year-old man without any symptoms or laboratory signs of anaemia. Sporadic erosions were found in another 2 healthy volunteers. Erosions are non-specific mucosal reaction on different etiopathogenetic factors (Crohn's disease, infectious enteritis, drug-induced damage etc.). The aetiology of these isolated erosions in our study was probably non-inflammatory as the surrounding mucosa was normal and they were no laboratory markers of systemic inflammatory reaction. The first erosion was observed in a 50-year-old woman in the distal ileum. This area may be prone to mechanical and ischaemic injury due to motility and repetitive constriction of ileo-caecal region. In the second case haemorhagic erosions in the proximal duodenum (postbulbar region) were found in a 34-year-old woman. The possible aetiology is questionable; it could be inbalace of aggressive and protective mucosal mechanisms. Another clinically non-significant findings observed in our study were suspected, tiny $(<3 \mathrm{~mm})$ intestinal polyps (in distal ileum explained as focal nodular lymphoid hyperplasia), with no malignant potential and no risk of complications and therefore with no need for further investigation or treatment. The only one identified submucosal tumour has the appearance of lipoma (yellowish translucent, smooth, circumscribed). Flebectasias, lymphangiectasias and xanthomas are frequently diagnosed during capsule enteroscopy and they need no specific treatment. Extra-intestinal findings can be an important part of capsule endoscopy in asymptomatic persons, potentially significant with possible further complications. Although capsule endoscopy is dedicated for the small bowel investigation, visualized parts of the oesophagus, stomach and caecum should be evaluated and all extra-intestinal findings should be described, too (16). The most frequent findings were erythematous and erosive gastropathy in our study. Caecal polyp diagnosed by means of capsule endoscopy was removed during subsequent colonoscopy. Small gastric polyps were identified as suspected fundic gland polyps with no need of treatment.

\section{Conclusions}

Significant part of healthy subjects had abnormal findings at wireless capsule endoscopy. These findings had no clinical relevance, as all these persons remained fully asymptomatic during a 36-month follow-up. Such an endoscopic appearance would be previously evaluated as "pathological". This is a principal report alerting that all findings of any control group of wireless capsule endoscopic studies must be evaluated with caution.

\section{Acknowledgements}

This study was supported by research project IGA NT 13532-4/2012 from the Ministry of Health, Czech Republic.

\section{References}

1. Iddan G, Meron G, Glukhovsky A, Swain P. Wireless capsule endoscopy. Nature 2000; 405(6785): 417

2. Tacheci I, Bradna P, Douda T, et al. NSAID-Induced Enteropathy in Rheumatoid Arthritis Patients with Chronic Occult Gastrointestinal Bleeding: A Prospective Capsule Endoscopy Study. Gastroenterol Res Pract 2013; 2013: 268382.

3. Ladas SD, Triantafyllou K, Spada C, et al. European Society of Gastrointestinal Endoscopy (ESGE): recommendations (2009) on clinical use of video capsule endoscopy to investigate small-bowel, esophageal and colonic diseases. Endoscopy 2010; 42(3): 220-7.

4. Tacheci I, Suchanek S, Drastich P, et al. Standard ČGS pro kapslovou endoskopii tenkého střeva. Gastroent Hepatol 2011; 65(4): 195-201.

5. Keuchel M, Hagenmuller F, Tajiri H. Video Capsule Endoscopy: A Reference Guide and Atlas. Berlin: Springer Verlag, 2014.

6. Tacheci I, et al. Kapslová endoskopie. Hradec Králové: Nucleus HK, 2008.

7. Morris AJ, Wasson LA, MacKenzie JF. Small bowel enteroscopy in undiagnosed gastrointestinal blood loss. Gut 1992; 33(7): 887-9.

8. Swain P, Fritscher-Ravens A. Role of video endoscopy in managing small bowel disease. Gut 2004; 53(12): 1866-75.

9. Buscaglia JM, Kapoor S, Clarke JO, et al. Enhanced diagnostic yield with prolonged small bowel transit time during capsule endoscopy. Int J Med Sci 2008; 5(6): 303-8.

10. Watier A, Devroede G, Perey B, Haddad H, Madarnas P, Grand-Maison P. Small 
erythematous mucosal plaques: an endoscopic sign of Crohn's disease. Gut 1980; 21(10): 835-839.

11. Graham DY, Opekun AR, Willingham FF, Qureshi WA. Visible small-intestina mucosal injury in chronic NSAID users. Clin Gastroenterol Hepatol 2005 Jan; 3(1): 55-9.

12. Maiden L, Thjodleifsson B, Theodors A, Gonzalez J, Bjarnason I. A quantitative analysis of NSAID-induced small bowel pathology by capsule enteroscopy. Gastroenterology 2005 May; 128(5): 1172-8.

13. Caunedo-Alvarez A, Gomez-Rodriguez BJ, Romero-Vazquez J, et al. Macroscopic small bowel mucosal injury caused by chronic nonsteroidal anti-inflammatory drugs (NSAID) use as assessed by capsule endoscopy. Rev Esp Enferm Dig 2010 Feb; 102(2): 80-5.

14. Goldstein JL, Eisen GM, Lewis B, et al. Small bowel mucosal injury is reduced in healthy subjects treated with celecoxib compared with ibuprofen plus omeprazole. as assessed by video capsule endoscopy. Aliment Pharmacol Ther 2007 May 15 25(10): 1211-22.

15. Maiden L, Thjodleifsson B, Seigal A, et al. Long-term effects of nonsteroidal anti-inflammatory drugs and cyclooxygenase- 2 selective agents on the small bowel: a cross-sectional capsule enteroscopy study. Clin Gastroenterol Hepatol 2007 Sep; 5(9): 1040-5.

16. Tacheci I, Deviere J, Kopacova M, Douda T, Bures J, Van Gossum A. The importance of upper gastrointestinal lesions detected with capsule endoscopy in patients with obscure digestive bleeding. Acta Gastroenterol Belg 2011 Sep; 74(3): 395-9.

Received: 17/05/2016

Accepted: 09/06/2016 